\title{
Persuading Visual Attention through Geometry
}

\author{
Youngmin Kim, Student Member, IEEE, and Amitabh Varshney, Member, IEEE
}

\begin{abstract}
Artists, illustrators, photographers, and cinematographers have long used the principles of contrast and composition to guide visual attention. In this paper, we introduce geometry modification as a tool to persuasively direct visual attention. We build upon recent advances in mesh saliency to develop techniques to alter geometry to elicit greater visual attention. Eye-tracking-based user studies show that our approach successfully guides user attention in a statistically significant manner. Our approach operates directly on geometry and, therefore, produces view-independent results that can be used with existing view-dependent techniques of visual persuasion.
\end{abstract}

Index Terms-Mesh saliency, mesh filtering, smoothing, enhancement, eye tracking, viewer attention, visual persuasion.

\section{INTRODUCTION}

$\mathrm{M}$ UCH progress has been made in the arts in establishing a visual narrative that guides a viewer's attention in a mild manner through a scene. In this paper, we explore how one can use techniques based on mesh processing to persuade a viewer to look at certain regions more than others. We establish a relationship between geometry modification and viewer attention. We quantify human attention by measuring the eye movements of subjects as they examine meshes altered by our technique.

Traditional art, illustration, and photography have long explored the principles of visual persuasion. Most of these principles, such as those based on camera or illumination, are view dependent. However, in an interactive medium such as 3D graphics, we believe that there is an additional need for persuading visual attention through view-independent attributes such as geometry filtering. The advantage of pursuing visual attention persuasion through geometry manipulation is that, by pushing the influence of attention deeper into the graphics pipeline, content creators can have greater flexibility in using other conventional techniques later in the graphics pipeline.

The main contributions of this paper are the following:

1. We introduce the concept of persuading visual attention through geometry modification.

2. We present a general class of mesh filters that can increase the saliency of a mesh over an arbitrary region of interest.

3. We are the first to present a view-independent method of visual attention persuasion that directly operates on meshes.

4. We examine the effectiveness of persuading visual attention through geometry using an eye-trackingbased user study.

- The authors are with the Department of Computer Science and UMIACS, University of Maryland, College Park, 4406 A.V. Williams Building, College Park, MD 20742. E-mail: \{ymkim, varshney\}@cs.umd.edu.

Manuscript received 29 May 2007; revised 10 Oct. 2007; accepted 12 Oct. 2007; published online 31 Oct. 2007.

For information on obtaining reprints of this article, please send e-mail to: tvcg@computer.org and reference IEEECS Log Number TVCG-2007-05-0057. Digital Object Identifier no. 10.1109/TVCG.2007.70624.
5. We perform a wide range of statistical analyses to show that our technique is more effective in drawing and holding the viewers' attention than prior art (the Gaussian-based enhancement method).

\section{BACKGROUND}

Artists have long used a rich collection of compositional and rendering techniques to persuade the viewers to pay greater attention to specific characters and objects in their paintings. The artist-determined visual hierarchy leads the viewer through the painting to see objects in the order of their relative importance, thereby finely controlling the communication of the message and purpose of their work. Image features such as luminance, color, and orientation are believed to guide the visual attention in low-level human vision [1], and the role of luminance and texture contrast in attracting visual attention has been recently verified by Parkhurst and Niebur [2]. Recently, ideas inspired by the principles of visual communication based on art, perceptual psychology, and cognitive science have begun to be carefully studied in the context of visual depiction [3]. As an example, discrepant lighting can be used to emphasize and depict a user-specified detail in images [4], as well as meshes [5], [6].

Visual attention is a complex process that consists of an observer processing selected aspects of visual information more than others [1]. Eye movements are one of the most important but not the only means of visual selection. Many models of visual attention and saliency in an image have been evaluated by their ability to predict eye movements [7], [8]. Several computational models of visual saliency that model human attention have been developed. Based on Koch and Ullman's [9] model of early vision, Itti et al. [10] identify regions of images that are distinct from their neighbors by applying center-surround mechanisms to different multiscale image feature maps and combine them to compute a saliency value for each pixel. There are a number of other approaches to modeling visual attention that combine local and global image features as in the work of Tsotsos et al. [11], Milanese et al. [12], Rosenholtz [13], and Torralba [14]. Santella and DeCarlo [15] have studied the relationship between eye movements and image 


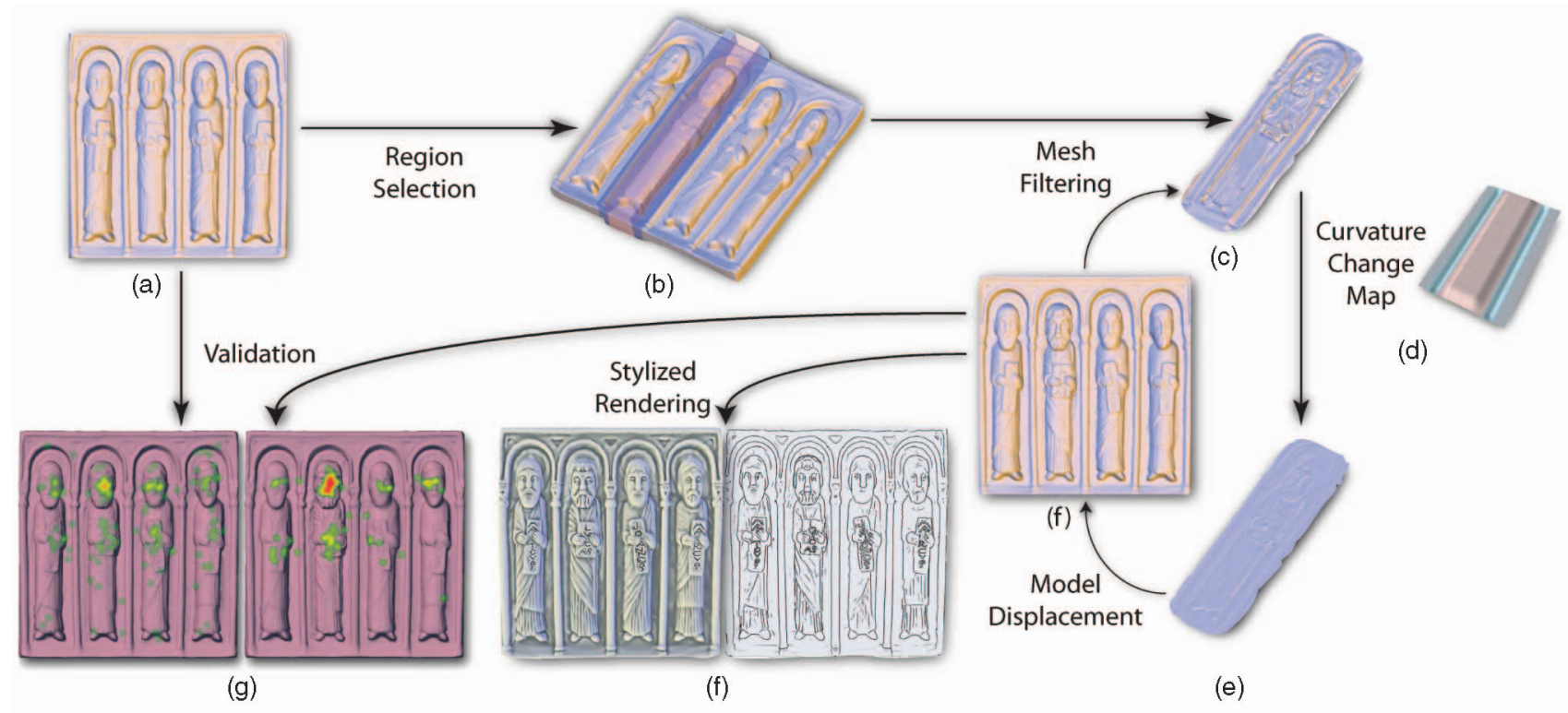

Fig. 1. An overview of persuading visual attention through geometry. The content creator defines a region of attention over the mesh in (a), as shown in (b). Mesh filtering over the desired attention region provides a set of displacements (c) along the vertex normals. Vertex displacements are weighted by a curvature change map (d) and then added to the input mesh. The resulting mesh in (f) elicits greater visual attention in the desired region (the second saint). Further, the mesh retains its visual attention persuasiveness through various rendering styles and illuminations (g), and this is validated by an eye-tracking-based user study, as shown in (h).

saliency to explore the effect of local detail modulation to the way viewers examine an image. Enns and Rensink [16] have shown that the salient pop-out phenomenon is not just limited to 2D image attributes but also occurs for 3D objects that differ in spatial orientation. Methods for computation of salient regions on 3D meshes have been developed by Watanabe and Belyaev [17], Hisada et al. [18], Lee et al. [19], and Howlett and O'Sullivan [20].

Saliency has been used in several 3D graphics applications, including animation compression [21], rendering acceleration [22], viewpoint selection [19], [23], shape matching [24], and mesh simplification [19], [20]. Perception-based methods have been used for level-of-detail simplification for 3D meshes [25], [26]. Su et al. [27] have developed an elegant postprocessing technique to reduce the saliency of distracting regions in an image. They alter regional saliency by reducing its texture variation through the use of steerable pyramids [28]. Kim and Varshney [29] have developed a saliency-based enhancement technique to draw visual attention to a user-specified region by changing the luminance and saturation in a direct volume rendering application. In this paper, we examine how saliency alteration in 3D geometry may affect viewer attention. We thereby aim to expand the scope of visual attention persuasion techniques from luminance contrast, color contrast, and texture contrast to include changes in geometry.

\section{Overview}

Visual attention can be guided in a goal-driven fashion or by external stimuli [30]. In this work, we focus on the latter and therefore do not consider the high-level semantics of the objects or tasks at hand. Visual attention can be drawn to a specific region by simply having the selected pixels rapidly change and flash colors. Other approaches to draw attention to a region could include lighting it brightly, using high-saturation colors, and adding a high-curvature spike. Although these approaches would likely work, they should be considered coercive and obtrusive instead of being persuasive. The challenge in gently guiding visual attention is to do so in a finely nuanced style that only introduces subtle changes. In this paper, we explore visual attention persuasion by making changes only to geometry. The various stages in our approach are shown in Fig. 1 and summarized below:

Region selection. We allow the content designer to specify attentional regions in a scene by directly selecting vertices, regions, or objects. The details of this interface are covered in Section 4.

Persuasion filters. In Section 5, we discuss an approach to enhance the saliency of a region to attract greater visual attention by designing a general class of mesh filters.

Validation. Once a persuasion filter has been applied to a given region, we would like to have some objective evidence that it results in eliciting greater visual attention. Eye tracking is the most prevalent method of estimating visual attention. We have conducted an eye-tracking-based user study to verify the impact of our persuasion filters and report the results in Section 6.

Stylized rendering. Our approach of geometry filtering incorporates visual attention persuasion early in the graphics pipeline. We have empirically observed that these changes are successfully propagated to the final rendering under several illumination and rendering styles. We discuss this in Section 6.4.

\section{Region Selection}

The input to our method is a mesh and a collection of one or more regions on it that have been selected by the user for 

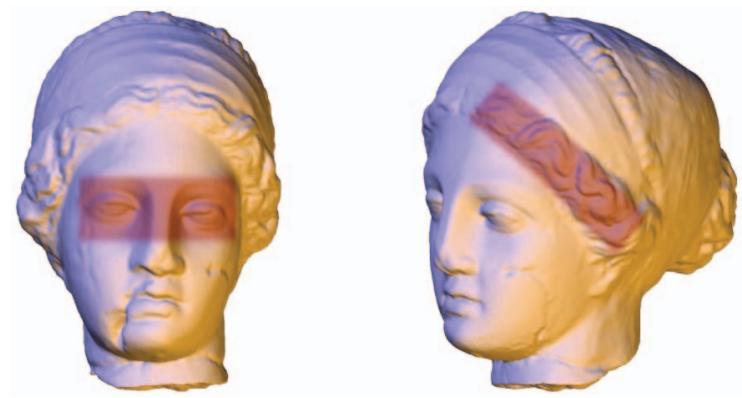

Fig. 2. A user can interactively define the regions on the mesh where greater attention is desired.

eliciting greater visual attention. One of the simplest and most effective ways to specify regions on a mesh is by a WYSIWYG painting interface such as the one developed by Hanrahan and Haeberli [31]. We use a similar screen-space brush in which the screen-space coordinates are projected back onto the surface by inverting the viewing transformation. This allows us to readily locate the appropriate mesh triangles and vertices in the vicinity of the brush location. The size of the brush is variable and can be modified to provide the user with coarse-to-fine control over the selected regions.

Although, initially, it seemed attractive to allow a user to paint arbitrarily shaped regions, we soon realized that this was not necessarily the best way to validate our results through user studies. The challenge here lay in attempting to isolate the effects of our persuasion filters from those arising naturally from the shape of the selected regions. Thus, if a user specifies a $T$-shaped region on a mesh, the confluence of two linear regions is likely to elicit greater attention due to perceptual principles other than the ones we are targeting in this paper. Therefore, we have only used simple circular or rectangular region selections. We have found it helpful to also add a spherical region selector. Fig. 2 shows some of the regions selected by our region selection tool.

\section{Persuasion Filters}

Modifying the contrast of visually important features has been recently used in several contexts such as video abstraction [32] and attention-based modulation of detail for exaggerated shading [6]. Winnemöeller et al. [32] regard the luminance and color opponency as visually important features and modified their contrast for image abstraction with the assumption that the changes in these frequencies are perceptually important.

We believe that manipulating geometry by smoothing and sharpening is a basic but useful method for guiding viewer attention in a view-independent way for 3D graphics applications. However, applying this approach to a 3D mesh is not trivial and has several issues. First, we have to identify which 3D mesh property to modify by smoothing and sharpening operators just as luminance and color opponency are used in imagery. Second, we need a framework that can structure the modification of the geometry in a controlled manner.
We change the mean curvature values at the vertices around the user-specified region by using bilateral displacements [33] and the magnitudes of bilateral displacements are guided by the mesh saliency. In this section, we explain our design choices.

\subsection{Bilateral Displacements}

Taubin [34] introduced the Laplacian-operator-based isotropic mesh smoothing. An implicit formulation for isotropic mesh smoothing based on the analogy of geometric flow was developed by Desbrun et al. [35]. Anisotropic filtering techniques were subsequently developed by Guskov et al. [36], Desbrun et al. [37], Zhang and Fiume [38], Bajaj and Xu [39], and others. Bilateral filtering was introduced for meshes by Fleishman et al. [33] and Jones et al. [40] as a more stable alternative to anisotropic diffusion. These approaches are similar in formulation but differ in how they predict the local surface around a point.

There are a number of methods to alter the mean curvature values of a mesh. These include applying any kind of smoothing and sharpening operators explained above, displacing vertices, changing local normals, and even applying level-of-detail techniques. Among these, we would like to focus on methods that change the mean curvature values in a controlled manner.

Nealen et al. [41] captured high-frequency details at a vertex $\mathbf{v}_{\mathbf{i}}$ using the Laplacian coordinates [35], [42] in their mesh editing framework as

$$
\mathbf{d}_{\mathbf{i}}=\mathbf{v}_{\mathbf{i}}-\frac{\sum_{\{i, j\} \in E} w_{i j} \mathbf{v}_{\mathbf{j}}}{\sum_{\{i, j\} \in E} w_{i j}} .
$$

Here, the vertices $\mathbf{v}_{\mathbf{j}}$ are the one-ring neighbors of a vertex $\mathbf{v}_{\mathbf{i}}$, and $w_{i j}$ are determined using the cotangent weights [42]. Since $d_{i}$ is in the local normal direction and the length $\left\|d_{i}\right\|$ is proportional to the mean curvature around vertex $\mathbf{v}_{\mathbf{i}}$, Laplacian-based vertex displacement offers a direct way to change the mean curvature values in the selected region of interest. However, the vertex displacement $\mathbf{d}_{\mathbf{i}}$ in (1) only operates for one-ring neighbors. We would like to guide the magnitudes of displacements by mesh saliency. Since the mesh saliency operator does not operate at one-ring neighbors but at a scale $\sigma$, we generalize the definition of neighbors with respect to the scale $\sigma$. Displacing vertices based on Fleishman et al.'s bilateral filter [33] satisfies this requirement while preserving all the benefits of Laplacian-based vertex displacement in changing mean curvatures. They smooth vertex $\mathbf{v}$ with a normal $\mathbf{n}$ as

$$
B(\mathbf{v})=\mathbf{v}+d \cdot \mathbf{n},
$$

$d=\frac{\sum_{\mathbf{p} \in N\left(\mathbf{v}, 2 \sigma_{c}\right)} W_{c}(\|\mathbf{v}-\mathbf{p}\|) W_{f}(<\mathbf{n}, \mathbf{v}-\mathbf{p}>)<\mathbf{n}, \mathbf{v}-\mathbf{p}>}{\sum_{\mathbf{p} \in N\left(\mathbf{v}, 2 \sigma_{c}\right)} W_{c}(\|\mathbf{v}-\mathbf{p}\|) W_{f}(<\mathbf{n}, \mathbf{v}-\mathbf{p}>)}$.

Here, $W_{c}(x)=e^{-x^{2} / 2 \sigma_{c}{ }^{2}}$ is the closeness smoothing function with parameter $\sigma_{c}$ that gives a greater weight to the vertices closer to the center vertex $\mathbf{v}, W_{f}(x)=e^{-x^{2} / 2 \sigma_{f}{ }^{2}}$ is the feature weight function with parameter $\sigma_{f}$ that penalizes a large variation in height from the local tangent plane, and $N\left(\mathbf{v}, 2 \sigma_{c}\right)$ is the neighborhood of $\mathbf{v}$ containing 
all vertices $\mathbf{p}$ such that $\|\mathbf{v}-\mathbf{p}\|<2 \sigma_{c}$. Note the similarities and differences between (1) and (2).

\subsection{Saliency-Guided Attention Persuasion}

Lee et al. [19] have defined the mesh saliency of a vertex $\mathbf{v}$ at a scale $\sigma$ using the center-surround mechanism as

$$
\mathcal{S}(\mathbf{v})=|G(\mathcal{C}, \mathbf{v}, \sigma)-G(\mathcal{C}, \mathbf{v}, 2 \sigma)|,
$$

where $\mathcal{C}$ denotes the mean curvature values around a vertex $\mathbf{v}$ and $G(\mathcal{C}, \mathbf{v}, \sigma)$ is the Gaussian-weighted average of the mean curvature of vertices in the neighborhood $N(\mathbf{v}, 2 \sigma)$. In fact, the center-surround mechanism used in this definition is the Difference of Gaussians $(D o G)$ function at a fine scale $\sigma$ and a coarse scale $2 \sigma$. We are interested in changing the mean curvature values at the vertices around the user-specified region of interest so that those modifications result in user-desired saliency changes $\Delta \mathcal{S}$. For this purpose, we slightly modify the center-surround mechanism and define the center-surround operator at a vertex $\mathbf{v}$ using the Laplacian of the Gaussian-weighted averages as

$$
\mathcal{S}(\mathbf{v})=w_{1} G(\mathcal{C}, \mathbf{v}, \sigma)-w_{2} G(\mathcal{C}, \mathbf{v}, 2 \sigma),
$$

where $w_{1}$ and $w_{2}$ indicate the positive weights of the Gaussian-weighted averages at a fine and a coarse scale, respectively. From this definition, the saliency change at a vertex $\mathbf{v}$ can be simply expressed as

$$
\Delta \mathcal{S}(\mathbf{v})=w_{1} G(\Delta \mathcal{C}, \mathbf{v}, \sigma)-w_{2} G(\Delta \mathcal{C}, \mathbf{v}, 2 \sigma),
$$

where $\Delta \mathcal{C}$ is defined as the curvature change map. Given a user-specified saliency change map $\Delta \mathcal{S}$, we can compute the curvature change map $(\Delta \mathcal{C})$ around a vertex $\mathbf{v}$ by solving the following system of linear equations:

$$
\left[\begin{array}{cccc}
c_{1,1} & c_{1,2} & \ldots & c_{1, n} \\
c_{2,1} & c_{2,2} & \ldots & c_{2, n} \\
\vdots & \vdots & \ddots & \vdots \\
c_{n, 1} & c_{n, 2} & \ldots & c_{n, n}
\end{array}\right]\left[\begin{array}{c}
\Delta \mathcal{C}\left(v_{1}\right) \\
\Delta \mathcal{C}\left(v_{2}\right) \\
\vdots \\
\Delta \mathcal{C}\left(v_{n}\right)
\end{array}\right]=\left[\begin{array}{c}
\Delta \mathcal{S}\left(v_{1}\right) \\
\Delta \mathcal{S}\left(v_{2}\right) \\
\vdots \\
\Delta \mathcal{S}\left(v_{n}\right)
\end{array}\right],
$$

where the coefficients $c_{i, j}$ represent the difference between two Gaussian weights at scale $\sigma$ and at scale $2 \sigma$ for a vertex $v_{j}$ in the neighborhood of the center vertex $v_{i}$. Setting the two weights $w_{1}$ and $w_{2}$ to be equal results in a rankdeficient system. We have observed that the system is stable for unequal weights. This corresponds to defining the saliency function using an aggregate of DoG and a Gaussian $(G)$ instead of just a DoG function. Specifically, we have found that using the weights $w_{1}=3 / 4$ and $w_{2}=1 / 4$, which corresponds to $1 / 4 D o G+1 / 2 G$, results in a stable system that alleviates the rank deficiency of the coefficient matrix.

We solve this system of linear equations at multiple scales $\sigma_{i}$ to get the curvature change map $\left(\Delta \mathcal{C}_{i}\right)$ at each scale $\sigma_{i}$. The overall curvature change map is computed as the multiscale summation of $\Delta \mathcal{C}_{i}$. Assuming a spherical region of interest, the resulting curvature change map has the shape of the green curve shown in Fig. 3a.

Since we are interested in emphasizing user-specified regions on meshes, we generalize the spherical region of interest by using distance fields on a mesh [43]. Given a region of interest $(R O I)$, we compute the distance field from the boundary of $R O I$ and define the radius of the

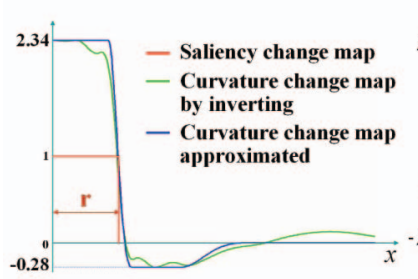

(a)

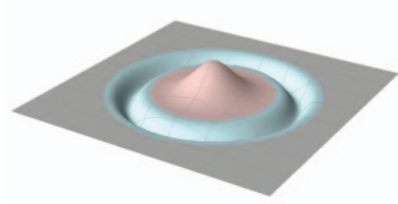

(c)

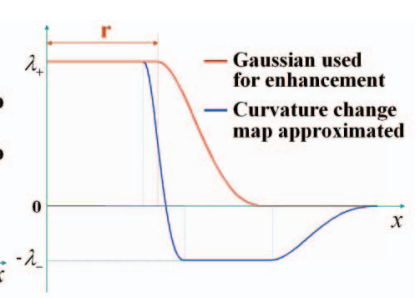

(b)

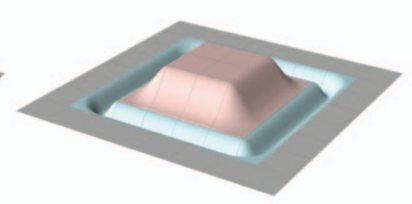

(d)
Fig. 3. Saliency-guided curvature change map. Here, $r$ denotes the radius of the user-specified region. (a) The curvature change map generated by the multiscale summation of curvature change maps is shown in green, and the approximation of it is shown in blue with $\lambda_{+}=$ 2.34 and $\lambda_{-}=0.28$ for comparison. (b) The Gaussian that is used for enhancement in the user study in Section 6. (c) and (d) The curvature change maps for circular and rectangular regions.

user-specified region $r$ as the distance from the innermost point to the boundary. Let $d_{b}(\mathbf{v})$ be the distance from the boundary of $R O I$ to the vertex $\mathbf{v}$. We define $\operatorname{dist}(\mathbf{v})$ as $r+$ $d_{b}(\mathbf{v})$ for the vertex $\mathbf{v}$ that is outside of $R O I$ and $r-d_{b}(\mathbf{v})$ for the vertex $\mathbf{v}$ that is inside of ROI. The resulting curvature change map based on this distance field is shown for a rectangular region in Fig. 1d and Fig. 3d.

We change the mean curvature values of the vertices in a mesh by using the bilateral displacements. We modify the mean curvature around a vertex $\mathbf{v}$ by displacing it as

$$
\mathcal{P}(\mathbf{v})=\mathbf{v}-\Delta \mathcal{C}(\operatorname{dist}(\mathbf{v})) \cdot d \cdot \mathbf{n},
$$

where $d$ is the displacement in the normal direction $\mathbf{n}$ for vertex $\mathbf{v}$ from the bilateral mesh filter in (2), and $\Delta \mathcal{C}(x)$ is the curvature change map. We refer to this as the persuasion filter $\mathcal{P}$.

In practice, we approximate the computed curvature change map by piecewise $C^{2}$-continuous degree-4 polynomial radial functions inspired by [44] as

$$
\begin{aligned}
& \Delta \mathcal{C}(x)=d(x)+w(x) \cdot g(\rho(x), \sigma(x)), \\
& g(\rho, \sigma)= \begin{cases}\left(1-\frac{\rho}{2 \sigma}\right)^{3}\left(\frac{3 \rho}{2 \sigma}+1\right), & \text { if } \rho \leq 2 \sigma, \\
0, & \text { otherwise, }\end{cases}
\end{aligned}
$$

where $d(x), w(x), \rho(x)$, and $\sigma(x)$ are determined by

$$
\begin{cases}d=\lambda_{+}, w=0, & \text { if } x<\frac{7}{8} r, \\ d=-\lambda_{-}, w=\lambda_{+}+\lambda_{-}, \rho=x-\frac{7}{8} r, \sigma=\frac{3}{16} r, & \text { elif } x<\frac{10}{8} r, \\ d=-\lambda_{-}, w=0, & \text { elif } x<2 r \\ d=0, w=-\lambda_{-}, \rho=x-2 r, \sigma=\frac{1}{2} r, & \text { elif } x<3 r, \\ d=0, w=0, & \text { otherwise. }\end{cases}
$$

Our approximating function is shown by the blue curve in Fig. 3a. In our current implementation, we use $0.1 \leq \lambda_{-} \leq \lambda_{+} \leq 0.3$. For all the examples in this paper, we have applied the persuasion filter five times to the region of interest. 


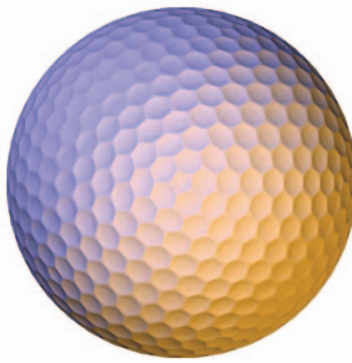

(a)

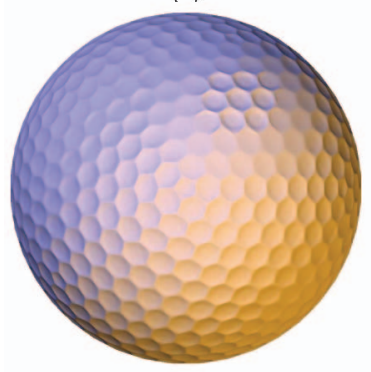

(c)

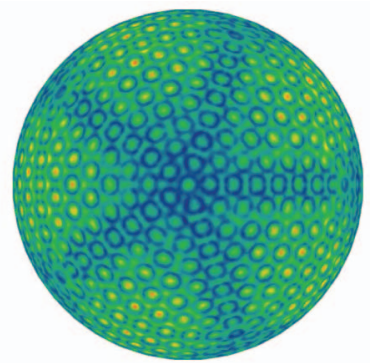

(b)

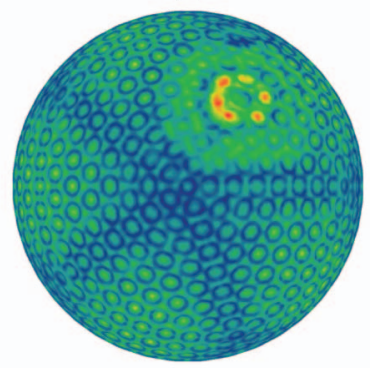

(d)
Fig. 4. Images show the saliency changes before and after the application of the persuasion filter on the Golf Ball model. (a) The original model. (b) Original saliency. (c) The result of applying the persuasion filters to the top part of the Golf Ball with $\left(\lambda_{+}=0.2, \lambda_{-}=0.2\right)$. (d) Saliency of the result in (c).

Fig. 3b shows the Gaussian fall-off function used for the comparisons in Section 6. The Gaussian fall-off function has been used in regional enhancement for volume illustration [45], in attention-based modulation of detail for exaggerated shading [6], and in stylized rendering [46]. There are several ways to define the Gaussian fall-off function. In this paper, we have used the Gaussian fall-off from the boundary of the region of interest instead of from a point to more closely match our computed curvature change map. We call the filter based on this Gaussian fall-off function the Gaussian filter. For our evaluations, we have kept the maximum change $\left(\lambda_{+}\right)$of the Gaussian filter to be the same as the maximum change of the approximated curvature change map in blue in Fig. 3b, and the standard-deviation $(\sigma)$ of this function from the boundary of the region of interest is determined to make the area under its curve equal to that under the persuasion filter curve (to equalize the changes introduced by the two filters).

Fig. 4 shows the application of the persuasion filter around the front part of the Golf Ball model, which results in an average saliency increase of 101.6 percent for the region.

Fig. 8c further shows the enhancement by persuasion filters. Compared to the original models in Fig. 8a, the effects of applying our filters are clearly visible. The application of the persuasion filter depends on the number of vertices in the region of interest. For the lower left part of the Golf Ball model in Fig. 8, 11.2K vertices were involved and it took 0.8 seconds. For one of the saints in Fig. 8, 26K vertices were involved and it took 2 seconds. When one of the armadillos in Fig. 8 was selected, there were $650 \mathrm{~K}$ vertices involved in the persuasion filtering, and it took about 15 seconds. These times were measured on a
2.93-GHz Intel(R) Core(TM)2 CPU Windows PC with 4 Gbytes of RAM.

\section{Validation and Results}

As we discussed in the previous section, the geometrybased model of visual attention persuasion results in an increase in mesh saliency over the user-specified region. The real measure of success, however, is whether this actually results in eliciting greater viewer attention. To gather objective evidence of the effectiveness of our approach, we have carried out an eye-tracking-based user study and have analyzed the results in two different ways: percentage of fixation points on the region of interest and average duration of consecutive fixation points on the region of interest. The first analysis is to see if our technique attracts the viewers' gaze to the region of interest, whereas the second analysis reveals if it holds the viewers' gaze. An excellent guide to the experimental design of eye-tracking-based user studies appears in the work of Parkhurst et al. [7].

\subsection{Hypotheses}

There are two hypotheses for each analysis, which result in a total of four hypotheses. Our first hypothesis (H1) is that the eye fixations increase over the region of interest by using the persuasion filter as compared to the original unaltered model. Our second hypothesis (H2) is that the eye fixations increase over the region of interest by using the persuasion filter compared to the use of a Gaussian filter. Our third hypothesis $(\mathrm{H} 3)$ is that the average durations of eye fixations on the region of interest increases by using the persuasion filter as compared to the original unaltered model. Our fourth hypothesis (H4) is that the average duration of eye fixations on the region of interest increases by using the persuasion filter compared to a Gaussian filter. We next examine the validity of these hypotheses. We examine hypotheses $\mathrm{H} 1$ and $\mathrm{H} 2$ in Section 6.3.1 and hypotheses H3 and H4 in Section 6.3.2.

\subsection{Experimental Design}

Subjects. Data were collected from a total of 18 subjects participating for pay. They had normal or corrected-tonormal vision and were not familiar with the goals of this study. Subjects were told to freely view the images with no assigned goal.

Physical setup. We have used the ISCAN ETL-500 eye tracker, which can record eye movements continuously at $60 \mathrm{~Hz}$. The study was carried out on a 17-inch LCD display with a resolution of $1,280 \times 1,024$, placed at a distance of 24 inches, subtending a visual angle of approximately 31.4 degrees horizontally. The subjects had a chin rest to minimize head movements and to maintain calibration. Our experimental setup is shown in Fig. 5.

Eye-tracker calibration. The standard calibration of ETL500 eye tracker was performed with four corner points and one center point. However, this was not sufficiently accurate for our purposes due to nonlinearities in the eyetracker-calibrated screen space. Therefore, we used the second calibration step, which involves a more densely sampled calibration phase similar to that in [7] with 


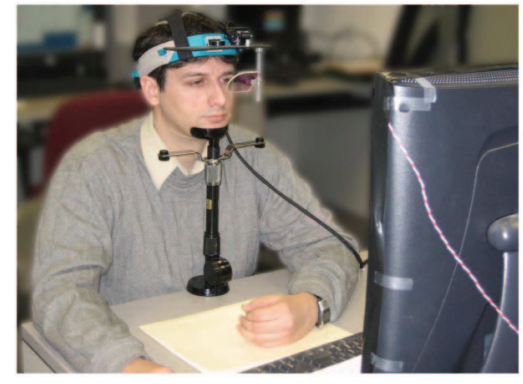

Fig. 5. Our experimental setup for the user study with the ISCAN ETL500 eye tracker.

13 additional points. For this, we asked the subjects to successively look at and click on 13 points presented on the screen. This gave us an accurate correspondence between the eye-tracker space and the monitor space for that subject. After this, we tested the accuracy of the calibration by asking the subjects to look at 16 randomly selected points on the screen. Of the 21 subjects, 18 were able to successfully calibrate to within an accuracy of 30 pixels (about 0.75 degree) for each of the 16 points. We proceeded with our study using these 18 subjects.

Duration. The user study had 13 trials (images) including four irrelevant images. Each trial started with the subject seeing a blank screen with a cross at the center of the screen. The subject was asked to look at the cross before clicking the mouse to bring up the next image. This ensured that each trial started with the subject's eyes fixated at the center of the image. Each image was shown for 5 seconds. Each study took about 80 seconds. Two irrelevant images were shown at the start of the experiment to give the subject a sense of the duration.

Image ordering. There were a total of 15 images used over all the experiments. Each image set consists of one original image and four filtered images in which one of the two regions is enhanced by either a Gaussian or our persuasion filter. The images and the regions of interest were carefully chosen so that the two regions of interest in each image set have similar shape, size, and saliency values (as computed in [19]). We have used the Golf Ball, the Romanesque Relief, and the Armadillos models shown in Fig. 8 for our study. Each user saw nine images out of these 15 images. When we ordered the images for each user, we considered differential carryover effects and the counterbalancing problem. First, we placed similar images far apart to alleviate differential carryover. At the same time, we did not place similar images in a perfectly regular manner so that a user could not predict the next image. We did this by inserting a couple of unrelated images. Alleviating differential carryover effects had the highest priority in our ordering because each user looked at three similar images (original and filtered with two different techniques). Finally, we randomized the order of regions and the order of enhancement types (Gaussian and persuasion filtered) to counterbalance overall effects.

Fixation points. We divide the data points from the eye tracker into two groups-fixation points, which correspond to a user looking at a single location, and saccade points, which correspond to fast eye movements from one fixation

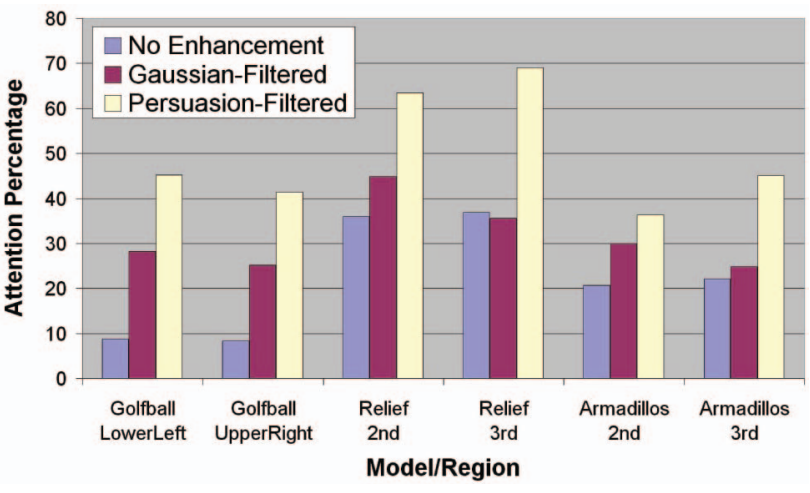

Fig. 6. The bars show the average percentage of fixation points on the region of interest for the original, the Gaussian-filtered, and the persuasion-filtered models.

point to the next. We followed an approach similar to the one suggested by Stampe [47] to identify fixations and saccades. We considered data points that had a velocity greater than 15 degrees/second as saccade points and removed them. We then averaged consecutive eye locations that were within 15 pixels and classified them as a single fixation point. Some researchers have advocated discarding short (exploratory) fixations in measuring the attention of the viewer [48]. We ignored the brief fixations below the threshold of $133 \mathrm{~ms}$. This corresponds to eight consecutive points in the ISCAN ETL-500 eye-tracking device. After we removed the short fixations, we weighted the contribution of each fixation point by its duration to give more weight to longer fixations. From here on, when we refer to a fixation point, we imply a duration-weighted fixation point. For every image that we used in our study, we had a specified region of desired visual attention.

\subsection{Data Analysis}

\subsubsection{Percentage of Fixation Points}

The results of our study can be seen in Fig. 6, and they show the increase in fixation points on the regions selected by the user. Each grouping of bars in Fig. 6 is labeled by the object or region on which the filters were applied. Fig. 7 shows the increase in the number of fixation points on the attention area after the application of persuasion filters. In Fig. 7b, the third saint was processed with persuasion filters, and this

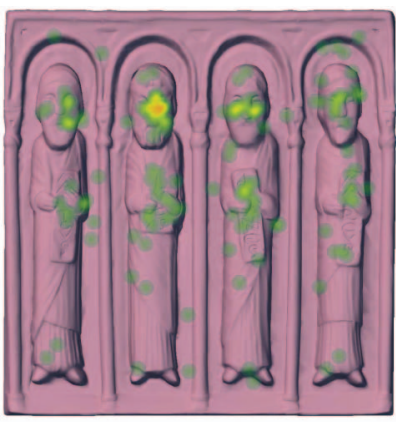

(a)

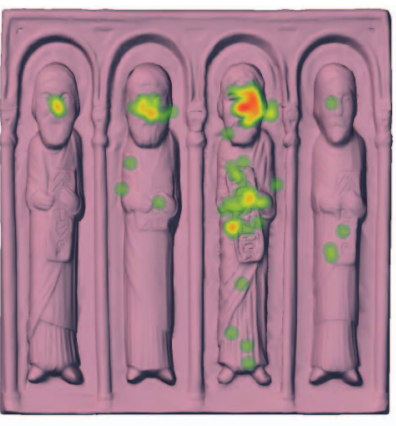

(b)
Fig. 7. (a) The fixation points on the original model. (b) The fixation points on the model altered by persuasion filters. Fixation points are recorded over the first 5 seconds from nine subjects and visualized with a hotspot map where warm colors show the areas of highest fixation count. 
(a)

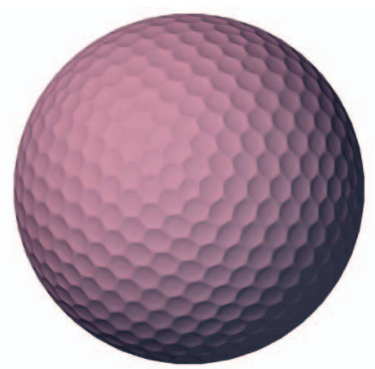

(b)

(c)
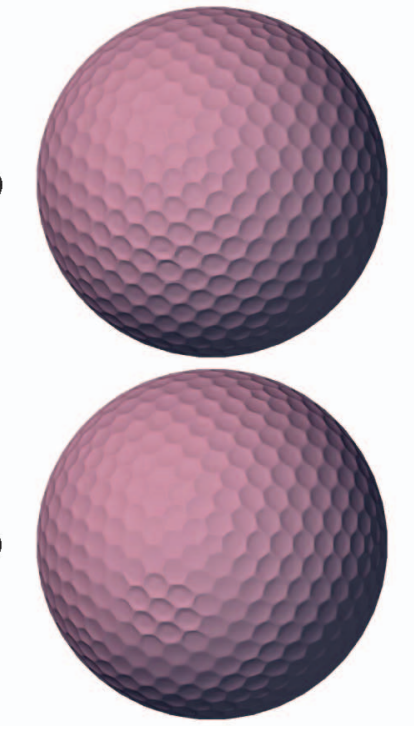
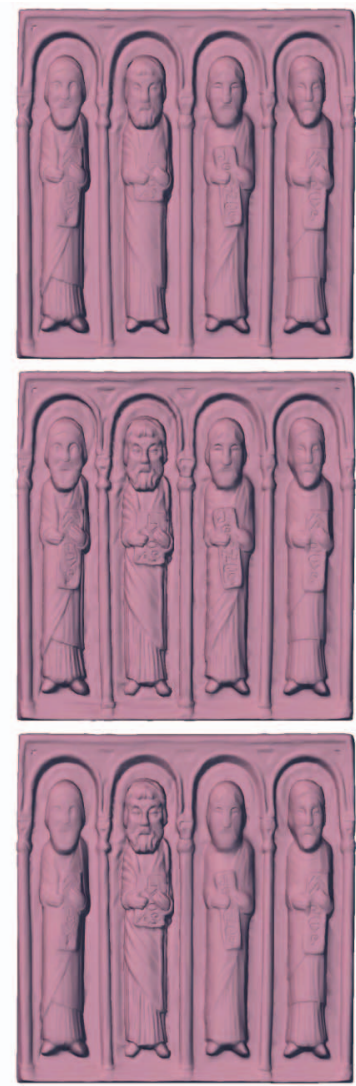
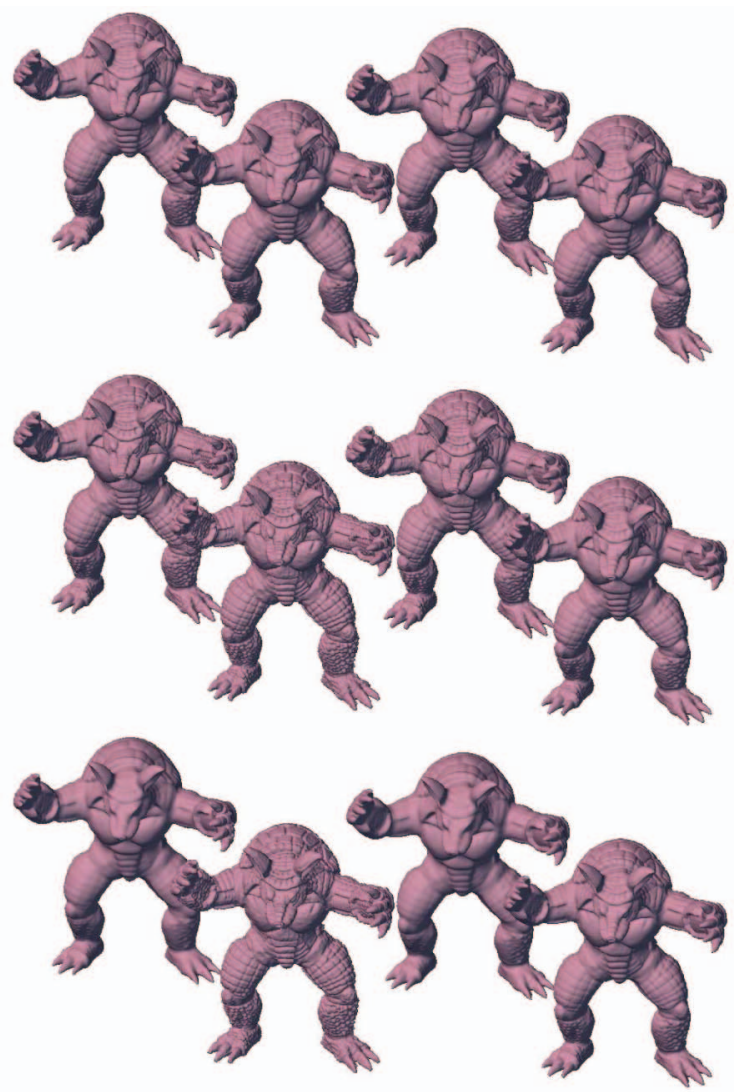

Fig. 8. A subset of the images used in the user study. (a) The original models. (b) Enhancement by the Gaussian filter. (c) Enhancement by the persuasion filter. Filters were applied to the lower left part of the Golf Ball model with $\left(\lambda_{+}=0.2, \lambda_{-}=0.2\right)$, the second saint in the Romanesque Relief model with $\left(\lambda_{+}=0.2, \lambda_{-}=0.1\right)$, and the second armadillo in the Armadillos model with $\left(\lambda_{+}=0.3, \lambda_{-}=0.3\right)$.

resulted in an 87 percent increase in the percentage of fixation points on it.

Table 1 shows the results of two-way ANOVA tests on the percentage of fixations with several different conditions. First, we carried out a two-way ANOVA for two conditions: filtering methods and image sets. As shown in Fig. 6, participants fixated more on the regions of interest when they are filtered with enhancement techniques. Overall, there was a strong difference in the percentage of fixations depending on enhancement techniques, $F(2,153)=30.09$, $p<0.001$.

Since we filtered two regions of interest for each image set, we did another analysis with filtering methods and regions for each image set. Among regions, there were no statistically significant differences $(F(2,48)=$ $0.027 \sim 0.195, p>0.661)$ as expected. For enhancement methods, there were statistically significant differences for all the models. We also carried out a two-way ANOVA test with two enhancement methods (Gaussian and

TABLE 1

The ANOVA Tests

\begin{tabular}{|l|c|c|}
\hline Condition & $F$-Value & $p$-Value \\
\hline Enhancement techniques on three sets & 30.09 & $<0.001$ \\
Enhancement techniques on Golfball & 13.45 & $<0.001$ \\
Enhancement techniques on Relief & 9.728 & $<0.001$ \\
Enhancement techniques on Armadillos & 7.2 & 0.002 \\
Gaussian vs. Persuasion on three sets & 21.23 & $<0.001$ \\
\hline
\end{tabular}

persuasion) and image sets to see if the persuasion-based enhancement is better. The result shows a significant improvement on the percentage of fixations with persuasion-based enhancement, $F(1,102)=21.23, p<0.001$.

Next, we performed a pairwise $t$-test on the percentage of fixations before and after we apply the enhancement techniques for each model (this is the only condition in the test). Table 2 shows the results for each of the models. We found a significant difference in the percentage of desired fixations after we applied the persuasion filters for each of the models. There was a significant difference in the percentage of desired fixations for the Gaussian filters only for the Golf Ball model. When we carried out a pairwise $t$-test between two filtering methods (Gaussian and persuasion) for each image set, we still observed a significant difference for each case.

TABLE 2

The Pairwise $t$-Tests (Two Tailed)

\begin{tabular}{|l|l|c|c|}
\hline Model & Condition & $t$-Value & $p$-Value \\
\hline Golfball & No Change vs. Gaussian & -3.34 & 0.004 \\
& No Change vs. Persuasion & -5.84 & $<0.001$ \\
& Gaussian vs. Persuasion & -2.81 & 0.012 \\
\hline Relief & No Change vs. Gaussian & -0.47 & 0.647 \\
& No Change vs. Persuasion & -4.69 & $<0.001$ \\
& Gaussian vs. Persuasion & -3.95 & 0.001 \\
\hline Armadillos & No Change vs. Gaussian & -1.56 & 0.138 \\
& No Change vs. Persuasion & -3.42 & 0.003 \\
& Gaussian vs. Persuasion & -2.31 & 0.033 \\
\hline
\end{tabular}


TABLE 3

The Average Duration and Standard Deviation of Fixation Points across the Subjects in Seconds

\begin{tabular}{|l|l|c|c|}
\hline Model & Condition & Average & Std Dev. \\
\hline Golfball & No Change & 0.204 & 0.270 \\
& Gaussian Enhancement & 0.374 & 0.351 \\
& Persuasion Enhancement & 0.744 & 0.467 \\
\hline Relief & No Change & 0.483 & 0.541 \\
& Gaussian Enhancement & 0.457 & 0.269 \\
& Persuasion Enhancement & 1.000 & 0.631 \\
\hline Armadillos & No Change & 0.408 & 0.318 \\
& Gaussian Enhancement & 0.476 & 0.354 \\
& Persuasion Enhancement & 0.678 & 0.618 \\
\hline
\end{tabular}

The results in this section clearly validate that there is a significant increase of fixations on the regions of interest by the persuasion filter over the original, as well as the Gaussian-filtered meshes.

\subsubsection{Average Duration of Consecutive Fixation Points}

Toward the end of Section 6.2, we discussed why we weighted the contribution of each fixation point by its duration. However, simply weighting fixation points by their duration cannot distinguish frequent brief fixations from a single long fixation. Henderson and Hollingworth [48] advocate that fixation points correlate with attention when they are longer in duration. To show that the images enhanced by our persuasion filter actually hold viewer gaze (draw attention), we have done another analysis on the average duration of fixation points on regions of interest. Table 3 shows the result of the average duration and their standard deviation of fixation points across the subjects for each model in seconds.

Since the standard deviations are large, we assessed the statistical significance of these results through ANOVA. We carried out a two-way ANOVA on the average durations for two conditions: enhancement methods and regions. Each image set has two regions of interest for a data set (Golf Ball, Relief, and Armadillos). Overall, there was a strong difference in the average durations depending on enhancement techniques, $F(2,153)=15.37, p<0.001$. Among regions, there were no statistically significant differences $(F(2,48)=0.492 .735, p>0.105)$, as expected.

We carried out a two-way ANOVA test comparing the unaltered (original) and persuasion-enhanced meshes with the two regions. The result shows a significant improvement on the average durations with persuasionbased enhancement, $F(1,102)=21.65, p<0.001$. We also carried out a two-way ANOVA test with two enhancement methods (Gaussian and persuasion) and the two regions. The result shows a significant improvement on the average durations with persuasion-based enhancement, $F(1,102)=16.96, p<0.001$.

The results validate the statistically significant increase of average durations on the regions of interest by the persuasion filter over the original, as well as the Gaussianbased methods.

\subsection{Stylized Rendering}

We have empirically tested our models with and without the application of the persuasion filters with several illumination and rendering styles. These include the standard OpenGL lighting model and suggestive contours [49]. The results of the application of our persuasion filters are clearly discernible with each of these lighting models and illumination styles. These are shown in Fig. 9. The reason for the successful propagation of fine geometry alterations to the final rendered image could be that the bilateral displacements allow us to preserve and enhance edges in the target attention area while smoothing them around it. These effects are perhaps most clearly visible in
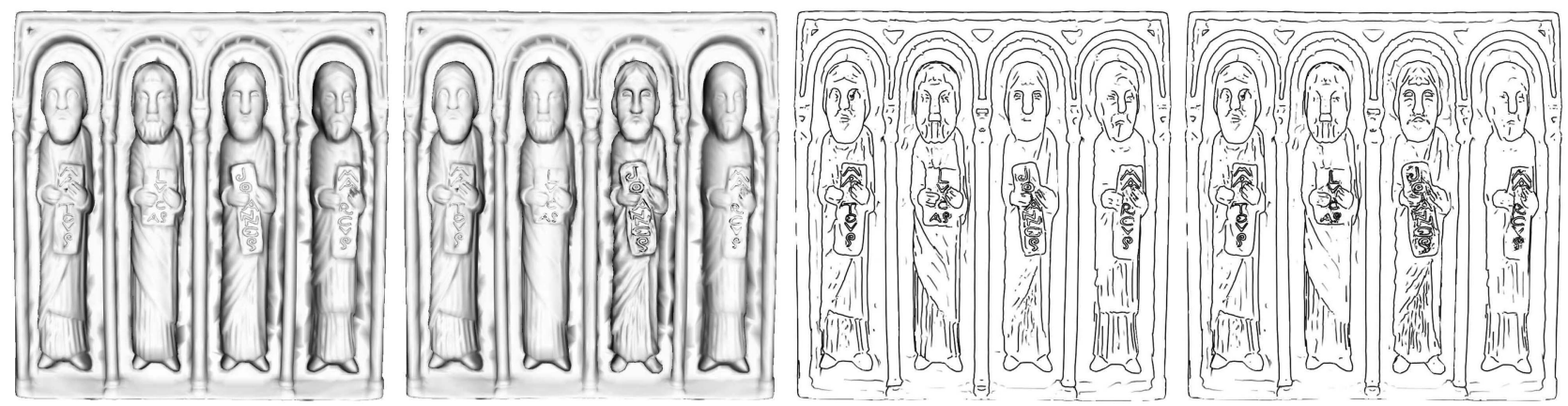

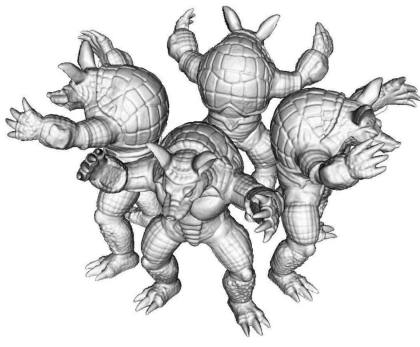

(a)

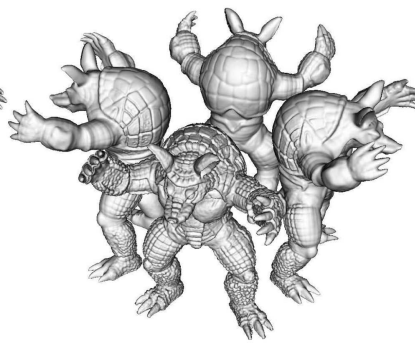

(b)

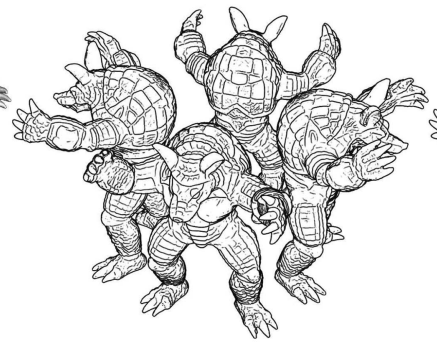

(c)

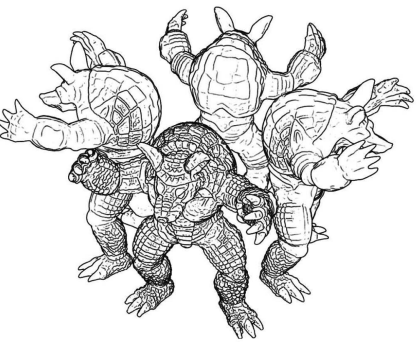

(d)

Fig. 9. Illustration of the Romanesque Relief and the Armadillos by Lambertian lighting and suggestive contours. (a) and (c) The original models. (b) Persuasion filters are applied to the third saint with $\left(\lambda_{+}=0.2, \lambda_{-}=0.1\right)$. (d) Persuasion filters are applied to the frontmost armadillo with $\left(\lambda_{+}=0.3, \lambda_{-}=0.3\right)$. 
the suggestive contour rendering emphasizing the third saint and the closest armadillo in Fig. 9.

Since persuasion filters are applied to the geometry before the graphics pipeline, this process is view independent. The empirical results in this section suggest that persuasion filters may also be able to elicit attention across a wide class of illumination and stylized rendering techniques. This encourages us to believe that it may be possible to combine geometry-based visual attention persuasion techniques with other visual attention persuasion techniques that are independent of geometry, such as luminance contrast, color contrast, and texture contrast.

\subsection{Discussion}

We believe that this work presents an exciting new direction in visual attention persuasion through geometry. At the same time, it is important to note the underlying assumptions, limitations, and distinctions from other related work.

In this paper, we have used bilateral displacements for smoothing and sharpening operations to alter the frequencies in the geometry. Therefore, if a surface is completely flat and its mean curvature is zero at every vertex, there is no enhancement by our persuasion filter since the displacement of the bilateral filter would be also zero. Also, we should note that sharpening is an inherently unstable process. We have tried to reduce these effects by using small delta values $(0.1 \sim 0.35)$ iteratively (five times) in our paper. However, the repeated application of our persuasion filter can eventually produce degenerate triangles. We are planning to incorporate remeshing [50], [51] and subdivision operators [52], [53] to incrementally remove degenerate triangles produced during the repeated application of our persuasion filter in the future.

Our desired saliency change map contains content at all frequencies, but our saliency operator, which has become a convolution, is band-pass. As a result, we use unequal weights $w_{1}$ and $w_{2}$ to reproduce all the frequencies of our saliency change map. Also, when we invert the saliency computation, we have only considered positive weights because we are interested in finding one assignment of the curvature change map that satisfies the saliency computation. Positive weights $w_{1}$ and $w_{2}$ emphasize the center and deemphasize the surrounding, whereas negative weights achieve the opposite. We believe that there could be an application that can benefit from deemphasizing the center and emphasizing the surrounding and plan to study the implications of the negative weights in the future.

Our technique is mainly designed to increase mesh saliency values around the selected region of interest. It could sometimes change the most salient regions in a mesh. The overall strength of such a change with respect to other salient features should be studied further. Also, we should note that, even though there is a qualitative saliency enhancement for the desired region, as in Fig. 4, we have not quantified the change in the presence of other salient regions on the mesh.

In this paper, we have provided a technique to guide viewer attention by only altering the geometry of the mesh and not its other appearance attributes. We did this because we wished to systematically establish the role of geometry alteration in persuading visual attention. Our method directly operates on meshes and therefore is more general than prior art that only operates at the level of rendering stylization. Further, our method is view independent, and therefore, by pushing the influence of attention deeper into the graphics pipeline, it allows content creators to have greater flexibility in using other conventional techniques later in the graphics pipeline. Our initial results in this direction have raised a number of other interesting questions and issues that we hope subsequent research will be able to address. For instance, we do not yet know if geometric techniques for persuading visual attention will prove to be more or less powerful than other visual attention persuasion techniques based on, say, luminance, chrominance, or texture contrast. How do these multiple channels of visual attention persuasion interact with each other's effectiveness-do they have mutual reinforcements or cancellations?

An important assumption for us has been to consider visual attention persuasion in a task-independent setting. This is similar in spirit to the early work in image saliency that relied on determining what constitutes the "pop-out" phenomenon during the highly parallel preattentive state of the human visual system. We have attempted to limit the role of semantics in three ways: 1) we presented each image for only 5 seconds, 2) we did not give any tasks to the users when viewing images, and 3) we had users view 3D models that were composed of semantically similar objects such as multiple armadillos and multiple saints. We hope that once geometric visual attention persuasion has been firmly established in a preattentive task-independent setting, it will lead to future work on semantically based techniques for persuading visual attention using geometry alteration.

Previous work on user-guided simplifications [54], [55] and spatially varying detail control [56] enables detail alteration based on user preferences or view dependence for rendering acceleration and for reducing visual clutter. Our work differs in its underlying motivation, as well as approach, in that we develop and validate a mechanism to guide viewer attention through geometry alteration.

Finally, we would like to mention that, although our filter as seen in Fig. 3 may appear to be similar at a first glance to the DoG filter, it is not; the classical DoG does not have a flat top.

\section{CONCLUSIONS AND FUtURE WORK}

Visual attention persuasion can be helpful in 3D graphics in several contexts-visually guiding users through complex graphics, facilitating interactions with attention-aware graphics tools and applications, and providing users with a more rewarding experience by guiding their attention to regions and objects desired by content creators. We have defined persuasion filters for meshes by inverting the center-surround saliency operator. Our user study shows that persuasion filters are able to draw greater user attention and that this condition is statistically significant. Our approach adds geometry alteration to the list of techniques at the disposal of visual persuaders.

At present, our filters are not informed by the local mesh properties. Kim and Rossignac [57] have recently 
developed a mesh filtering framework that can compute filter parameters based on user-specifiable mesh features. This is an interesting development that could lead to the design of persuasion filters that could be guided not just by regions but also by features. Another interesting direction to explore will be to incorporate view dependence in persuasion filtering. As mentioned earlier, in this work, we have exclusively considered the role of geometry in the context of visual attention persuasion. It will be interesting to see how other visual attention persuasion channels of color, luminance, and texture contrast interact with geometry alteration using the techniques of persuasion filters presented here.

\section{ACKNOWLEDGMENTS}

The authors gratefully acknowledge David Jacobs and François Guimbretière for several valuable discussions. Derek Juba, Hyunmo Kang, and Robert Patro have helped in greatly improving the presentation of ideas in this paper. They would also like to thank the anonymous reviewers for their detailed and highly constructive comments that were extremely helpful. The data sets used in this paper were provided by the Stanford Graphics Laboratory and the Princeton Graphics Group. This work was supported in part by US National Science Foundation Grants CCF 0541120, CCF 04-29753, CNS 04-03313, and IIS 04-14699.

\section{REFERENCES}

[1] S. Palmer, Vision Science: Photons to Phenomenology. MIT Press, 1999.

[2] D. Parkhurst and E. Niebur, "Texture Contrast Attracts Overt Visual Attention in Natural Scenes," European J. Neuroscience, vol. 3, no. 19, pp. 783-789, 2004.

[3] M. Agrawala and F. Durand, "Smart Depiction for Visual Communication," IEEE Computer Graphics and Applications, vol. 25, no. 3, pp. 20-21, 2005.

[4] D. Akers, F. Losasso, J. Klingner, M. Agrawala, J. Rick, and P. Hanrahan, "Conveying Shape and Features with Image-Based Relighting," Proc. IEEE Conf. Visualization (VIS '03), pp. 349-354, 2003.

[5] C.H. Lee, X. Hao, and A. Varshney, "Light Collages: Lighting Design for Effective Visualization," Proc. IEEE Conf. Visualization (VIS '04), pp. 281-288, 2004.

[6] S. Rusinkiewicz, M. Burns, and D. DeCarlo, "Exaggerated Shading for Depicting Shape and Detail," ACM Trans. Graphics (Proc. ACM SIGGRAPH '06), vol. 25, no. 3, pp. 1199-1205, 2006.

[7] D. Parkhurst, K. Law, and E. Niebur, "Modeling the Role of Salience in the Allocation of Overt Visual Selective Attention," Vision Research, vol. 42, no. 1, pp. 107-123, 2002.

[8] C.M. Privitera and L.W. Stark, "Algorithms for Defining Visual Regions-of-Interest: Comparison with Eye Fixations," IEEE Trans. Pattern Analysis and Machine Intelligence, vol. 22, no. 9, pp. 970-982, Sept. 2000.

[9] C. Koch and S. Ullman, "Shifts in Selective Visual Attention: Towards the Underlying Neural Circuitry," Human Neurobiology, vol. 4, pp. 219-227, 1985.

[10] L. Itti, C. Koch, and E. Niebur, "A Model of Saliency-Based Visual Attention for Rapid Scene Analysis," IEEE Trans. Pattern Analysis and Machine Intelligence, vol. 20, no. 11, pp. 1254-1259, Nov. 1998.

[11] J. Tsotsos, S. Culhane, W. Wai, Y. Lai, N. Davis, and F. Nuflo, "Modeling Visual-Attention via Selective Tuning," Artificial Intelligence, vol. 78, nos. 1-2, pp. 507-545, 1995.

[12] R. Milanese, H. Wechsler, S. Gil, J. Bost, and T. Pun, "Integration of Bottom-Up and Top-Down Cues for Visual Attention Using Non-Linear Relaxation," Proc. IEEE Conf. Computer Vision and Pattern Recognition (CVPR '94), pp. 781-785, 1994.
[13] R. Rosenholtz, "A Simple Saliency Model Predicts a Number of Motion Popout Phenomena," Vision Research, vol. 39, no. 19, pp. 3157-3163, 1999.

[14] A. Torralba, "Modeling Global Scene Factors in Attention," J. Optical Soc. of Am. A, vol. 20, no. 7, pp. 1407-1418, 2003.

[15] A. Santella and D. DeCarlo, "Visual Interest and NPR: An Evaluation and Manifesto," Proc. Third Int'l Symp. Non-Photorealistic Animation and Rendering (NPAR '04), pp. 71-150, 2004.

[16] J. Enns and R. Rensink, "Sensitivity to Three-Dimensional Orientation in Visual Search," Psychological Science, vol. 1, no. 5, pp. 323-326, 1990.

[17] K. Watanabe and A.G. Belyaev, "Detection of Salient Curvature Features on Polygonal Surfaces," Computer Graphics Forum (Proc. Eurographics '01), vol. 20, no. 3, pp. 385-392, 2001.

[18] M. Hisada, A.G. Belyaev, and T.L. Kunii, "A Skeleton-Based Approach for Detection of Perceptually Salient Features on Polygonal Surfaces," Computer Graphics Forum, vol. 21, no. 4, pp. $689-700,2002$.

[19] C.H. Lee, A. Varshney, and D. Jacobs, "Mesh Saliency," ACM Trans. Graphics (Proc. ACM SIGGRAPH '05), vol. 24, no. 3, pp. 659666, 2005.

[20] S. Howlett and C. O'Sullivan, "Predicting and Evaluating Saliency for Simplified Polygonal Models," ACM Trans. Applied Perception, vol. 2, no. 3, pp. 286-308, 2005.

[21] R. Mantiuk, K. Myszkowski, and S. Pattanaik, "Attention Guided MPEG Compression for Computer Animations," Proc. 19th Spring Conf. Computer Graphics (SCCG '03), pp. 239-244, 2003.

[22] H. Yee, S. Pattanaik, and D.P. Greenberg, "Spatiotemporal Sensitivity and Visual Attention for Efficient Rendering of Dynamic Environments," ACM Trans. Graphics, vol. 20, no. 1, pp. 39-65, 2001.

[23] H. Yamauchi, W. Saleem, S. Yoshizawa, Z. Karni, A. Belyaev, and H.-P. Seidel, "Towards Stable and Salient Multi-View Representation of 3D Shapes," Proc. IEEE Int'l Conf. Shape Modeling and Applications (SMI '06), pp. 265-270, 2006.

[24] R. Gal and D. Cohen-Or, "Salient Geometric Features for Partial Shape Matching and Similarity," ACM Trans. Graphics, vol. 25, no. 1, pp. 130-150, 2006.

[25] D.P. Luebke and B. Hallen, "Perceptually-Driven Simplification for Interactive Rendering," Proc. Eurographics Workshop Rendering Techniques, pp. 223-234, 2001.

[26] D. Luebke, M. Reddy, J. Cohen, A. Varshney, B. Watson, and R. Huebner, Level of Detail for 3D Graphics. Morgan Kaufmann, 2003.

[27] S.L. Su, F. Durand, and M. Agrawala, "De-Emphasis of Distracting Image Regions Using Texture Power Maps," Proc. Fourth IEEE Int'l Workshop Texture Analysis and Synthesis (Texture '05), pp. 119124, 2005.

[28] E.P. Simoncelli and W.T. Freeman, "The Steerable Pyramid: A Flexible Architecture for Multi-Scale Derivative Computation," Proc. Int'l Conf. Image Processing (ICIP '95), vol. 3, pp. 444-447, 1995.

[29] Y. Kim and A. Varshney, "Saliency-Guided Enhancement for Volume Visualization," IEEE Trans. Visualization and Computer Graphics, vol. 12, no. 5, pp. 925-932, Sept./Oct. 2006.

[30] M. Posner, "Orienting of Attention," Quarterly J. Experimental Psychology, vol. 32, pp. 3-25, 1980.

[31] P. Hanrahan and P. Haeberli, "Direct WYSIWYG Painting and Texturing on 3D Shapes," Proc. ACM SIGGRAPH, pp. 215-223, 1990.

[32] H. Winnemöeller, S.C. Olsen, and B. Gooch, "Real-Time Video Abstraction," ACM Trans. Graphics, vol. 25, no. 3, pp. 1221-1226, 2006.

[33] S. Fleishman, I. Drori, and D. Cohen-Or, "Bilateral Mesh Denoising," ACM Trans. Graphics (Proc. ACM SIGGRAPH '03), vol. 22, no. 3, pp. 950-953, 2003.

[34] G. Taubin, "A Signal Processing Approach to Fair Surface Design," Proc. ACM SIGGRAPH, pp. 351-358, 1995.

[35] M. Desbrun, M. Meyer, P. Schröder, and A. Barr, "Implicit Fairing of Irregular Meshes Using Diffusion and Curvature Flow," Proc. ACM SIGGRAPH, pp. 317-324, 1999.

[36] I. Guskov, W. Sweldens, and P. Schröder, "Multiresolution Signal Processing for Meshes," Proc. ACM SIGGRAPH, pp. 325-334, 1999.

[37] M. Desbrun, M. Meyer, P. Schröder, and A. Barr, "Anisotropic Feature-Preserving Denoising of Height Fields and Bivariate Data," Proc. Graphics Interface Conf. (GI '00), pp. 145-152, 2000.

[38] H. Zhang and E. Fiume, "Mesh Smoothing with Shape or Feature Preservation," Advances in Modeling, Animation, and Rendering: Proc. Computer Graphics Int'l Conf. (CGI '02), pp. 167-182, 2002. 
[39] C. Bajaj and G. Xu, "Anisotropic Diffusion of Surfaces and Functions on Surfaces," ACM Trans. Graphics, vol. 22, no. 1, pp. 432, 2003.

[40] T. Jones, F. Durand, and M. Desbrun, "Non-Iterative, FeaturePreserving Mesh Smoothing," ACM Trans. Graphics, vol. 22, no. 3, pp. 943-949, 2003.

[41] A. Nealen, O. Sorkine, M. Alexa, and D. Cohen-Or, "A SketchBased Interface for Detail-Preserving Mesh Editing," ACM Trans. Graphics (Proc. ACM SIGGRAPH '05), vol. 24, no. 3, pp. 1142-1147, 2005.

[42] M. Meyer, M. Desbrun, P. Schr, and A.H. Barr, "Discrete Differential-Geometry Operators for Triangulated 2-Manifolds," Visualization and Mathematics III, pp. 35-57, 2003.

[43] V. Surazhsky, T. Surazhsky, D. Kirsanov, S.J. Gortler, and H. Hoppe, "Fast Exact and Approximate Geodesics on Meshes," ACM Trans. Graphics (Proc. ACM SIGGRAPH '05), vol. 24, no. 3, pp. 553-560, 2005.

[44] H. Wendland, "Piecewise Polynomial, Positive Definite and Compactly Supported Radial Functions of Minimal Degree," Advances in Computational Math., vol. 4, no. 4, pp. 389-396, 1995.

[45] P. Rheingans and D. Ebert, "Volume Illustration: Nonphotorealistic Rendering of Volume Models," IEEE Trans. Visualization and Computer Graphics, vol. 7, no. 3, pp. 253-264, July-Sept. 2001.

[46] F. Cole, D. DeCarlo, A. Finkelstein, K. Kin, K. Morley, and A. Santella, "Directing Gaze in 3D Models with Stylized Focus," Proc. Eurographics Symp. Rendering, pp. 377-387, 2006.

[47] D. Stampe, "Heuristic Filtering and Reliable Calibration Methods for Video-Based Pupil Tracking Systems," Behavior Research Methods, Instruments and Computers, vol. 25, pp. 137-142, 1993.

[48] J.M. Henderson and A. Hollingworth, "Eye Movements during Scene Viewing: An Overview," Eye Guidance in Reading and Scene Perception, E.G. Underwood, ed., Elsevier Science, 1998.

[49] D. DeCarlo, A. Finkelstein, S. Rusinkiewicz, and A. Santella, "Suggestive Contours for Conveying Shape," ACM Trans. Graphics, vol. 22, no. 3, pp. 848-855, 2003.

[50] J. Vorsatz, C. Rössl, L. Kobbelt, and H.-P. Seidel, "Feature Sensitive Remeshing," Computer Graphics Forum, vol. 20, no. 3, pp. 393-401, 2001.

[51] V. Surazhsky and C. Gotsman, "Explicit Surface Remeshing," Proc. Eurographics/ACM SIGGRAPH Symp. Geometry Processing (SGP '03), pp. 20-30, 2003.

[52] D. Zorin, P. Schröder, and W. Sweldens, "Interpolating Subdivision for Meshes with Arbitrary Topology," Proc. ACM SIGGRAPH '96, pp. 189-192, 1996.

[53] N. Dyn, D. Levin, and J.A. Gregory, "A Butterfly Subdivision Scheme for Surface Interpolation with Tension Control," ACM Trans. Graphics, vol. 9, no. 2, pp. 160-169, 1990.

[54] G. Li and B. Watson, "Semi-Automatic Simplification," Proc. Symp. Interactive 3D Graphics (SI3D '01), pp. 43-48, 2001.

[55] Y. Kho and M. Garland, "User-Guided Simplification," Proc. Symp. Interactive 3D Graphics (SI3D '03), pp. 123-126, 2003.

[56] A. Ni, K. Jeong, S. Lee, and L. Markosian, "Multi-Scale Line Drawings from 3D Meshes," Proc. Symp. Interactive 3D Graphics (SI3D '06), pp. 133-137, 2006.

[57] B. Kim and J. Rossignac, "Geofilter: Geometric Selection of Mesh Filter Parameters," Computer Graphics Forum (Proc. Eurographics '05), vol. 24, no. 3, pp. 295-302, 2005.

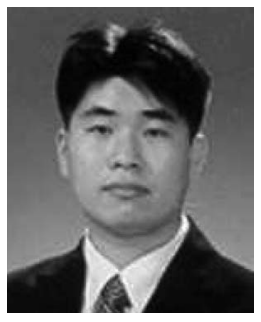

Youngmin Kim received the BS degree in computer engineering from Seoul National University, Seoul, in 1999 and the MS degree from the University of Maryland, College Park, in 2005. He is currently a PhD student of computer science at the University of Maryland, College Park. His research interests include 3D computer graphics, scientific visualization, high-performance computing, and visual perception. $\mathrm{He}$ is a student member of the IEEE.

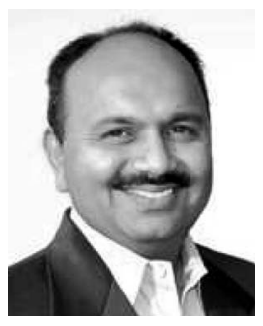

Amitabh Varshney received the BTech degree in computer science from the Indian Institute of Technology, Delhi, in 1989 and the MS and PhD degrees from the University of North Carolina, Chapel Hill, in 1991 and 1994. He is currently a professor of computer science at the University of Maryland, College Park. His current research interests are interactive 3D graphics, scientific visualization, geometric modeling, and molecular graphics. He received the US National Science Foundation's CAREER Award in 1995 and the IEEE Visualization Technical Achievement Award in 2004. He is a member of the IEEE and the IEEE Computer Society.

$\triangleright$ For more information on this or any other computing topic, please visit our Digital Library at www.computer.org/publications/dlib. 